\title{
LOCALLY ADVANCED BREAST CANCER
}

\author{
A Rodger, R C F Leonard, J M Dixon
}

\section{Clinical features of locally advanced breast cancer}

\section{Skin}

- Ulceration - Satellite nodules

- Dermal infiltration - Peau d'orange

- Erythema over tumour

Chest wall

Tumour fixation to

- Ribs

- Intercostal muscles

- Serratus anterior

Axillary nodes

- Nodes fixed to one another or to other structures
Locally advanced disease of the breast is characterised clinically by features suggesting infiltration of the skin or chest wall by tumour or matted involved axillary nodes. Large operable breast cancers and tumours fixed to muscle should not be considered as locally advanced. Depending on referral patterns and clinical definitions, between one in 12 and one in four patients with breast cancer present with locally advanced disease. Reflecting the differences in definition and the variable natural history of breast cancer, reported five year survival varies between $1 \%$ and $30 \%$. Median survival is about $2-2 \cdot 5$ years, which is similar to that described for breast cancer in the late 19 th and early 20 th centuries.

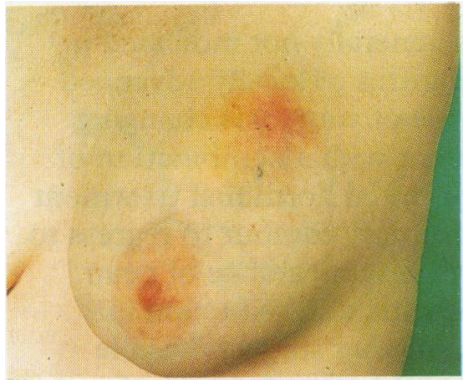

Inflammatory breast carcinoma.

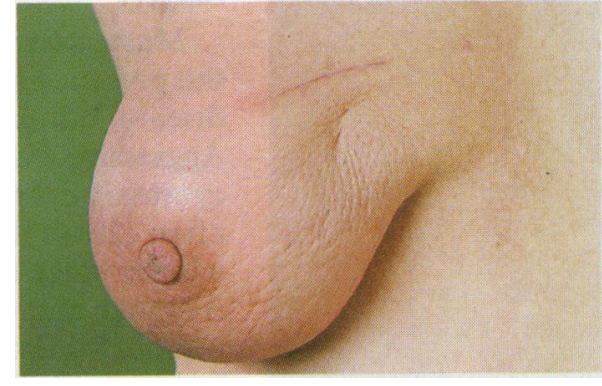

Peau d'orange associated with breast carcinoma.
Locally advanced breast cancer may arise because of its position in the breast (for example, peripheral), neglect (some patients do not present to hospital for month or years after they notice a mass), or biological aggressiveness (this includes all inflammatory cancers and most with peau d'orange). Inflammatory carcinomas are uncommon and are characterised by brawny, oedematous, indurated, and erythematous skin changes and have the worst prognosis of all locally advanced breast cancers.

\section{Treatment}

\section{Factors affecting choice of systemic treatment for locally advanced breast cancer}

\author{
Hormonal treatment \\ - Slow growing or indolent disease \\ - Oestrogen receptor positive cancer \\ - Elderly or unfit patients \\ Chemotherapy \\ - Inflammatory cancer \\ - Oestrogen receptor negative cancer \\ - Rapidly progressive cancer
}

Current treatments have had some impact on control of local disease but have had little overall effect on metastatic progression, although survival is better with hormone sensitive disease. Local and regional relapse is a major problem and affects more than half of patients.

\section{Role of systemic and local treatment}

The mainstay of local treatment has been radiotherapy. This is because surgery, generally mastectomy, results in high rates of local recurrence. In contrast, radiotherapy alone can produce high rates of local remission in both the breast and axilla, but with radiotherapy alone only $30 \%$ of patients remain free of locoregional disease at death. A combination of appropriate systemic treatment and radiotherapy can increase the initial rate of local response to over $80 \%$.

\section{Choice of systemic treatment}

Systemic treatment should be administered as part of a planned programme of combined systemic and local treatment. For frail patients treatment may initially be by tamoxifen, with radiotherapy held in reserve for relapse. 


\section{Choice of systemic treatment for locally advanced breast cancer}

\section{Hormonal treatment}

- Premenopausal women-ovarian ablation (surgery, radiation, or gonadotrophin releasing hormone antagonists)

- Postmenopausal women-tamoxifen

\section{Chemotherapy}

- Intravenous-infusion of fluorouracil combined with an anthracycline*

\section{- Intra-arterial}

*For example, doxorubicin, cyclophosphamide, and fluorouracil; or epirubicin, cisplatin, and fluorouracil.
Although standard chemotherapy regimens have increased rates of local control, they have had little impact on survival. Studies are currently under way to determine whether intensifying drug dosage (increasing the amount of drug given in a fixed period either by giving smaller doses more frequently or by combining higher doses with factors to encourage regeneration of bone marrow) does produce survival benefits. Current data suggest that infusional treatment with fluorouracil combined with the anthracyclines doxorubicin or epirubicin in regimens with cyclophosphamide or cisplatin produces higher response rates than intermittent regimens used for adjuvant chemotherapy.

\section{Radiotherapy for locally advanced breast cancer}

\section{Treatment areas}

- Breast Axilla and supraclavicular fossa

\section{Treatment}

- Megavoltage $x$ rays - Technique for enhancing skin dose

- 40-50 Gy in 15-25 fractions over 3-5 weeks

- Boost to tumour mass if possible by external beam or radioactive implant of $10-20 \mathrm{~Gy}$

Toxicity

- Lethargy - Skin erythema and small areas of moist desquamation

- Temporary mild dysphagia $\quad \bullet<3 \%$ risk of pneumonitis
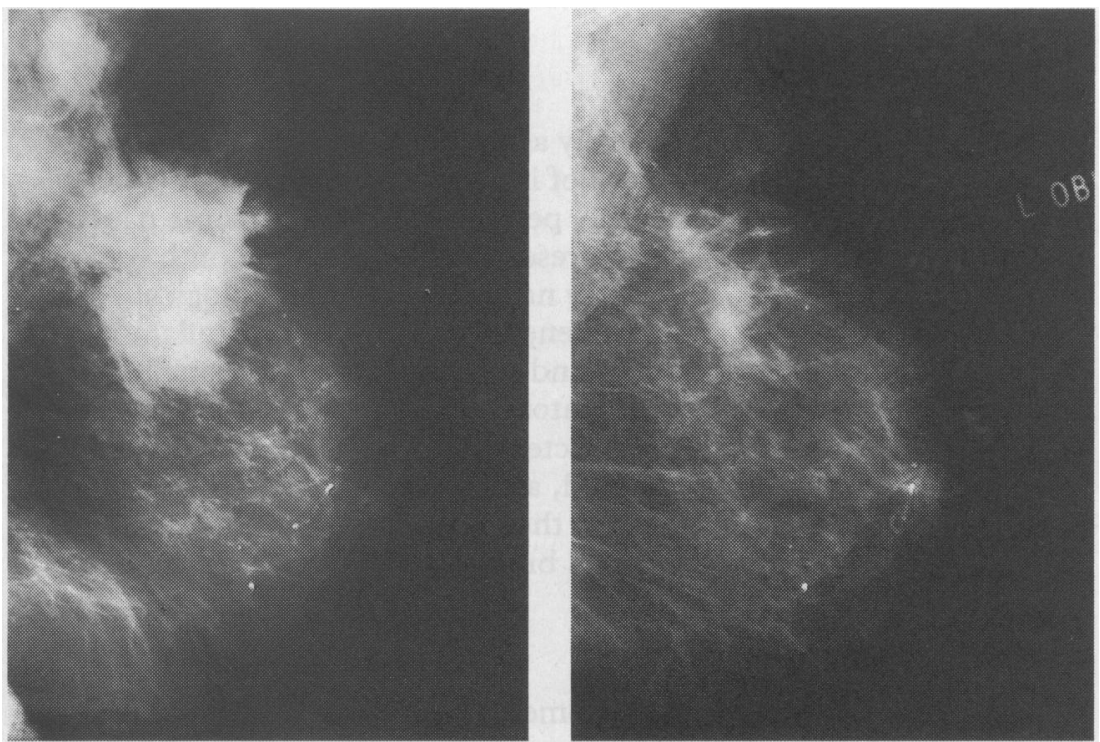

Mammogram of locally advanced breast tumour (left); and after chemotherapy, showing substantial reduction in tumour volume (right). (Tumour was operable after treatment.)

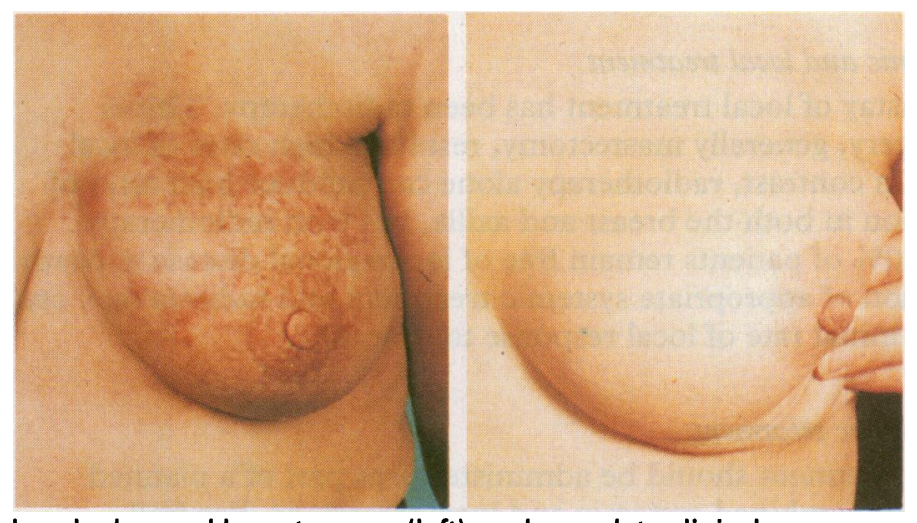

Local advanced breast cancer (left); and complete clinical response after chemotherapy (right).

\section{Radiotherapy}

Radiotherapy is generally well tolerated, even by elderly and frail patients. It can be given concurrently with systemic hormonal treatment, or it can be given after a course of primary chemotherapy to patients whose breast cancer still shows signs of local advancement. The breast skin requires full dose, and this will result in temporary erythema and possible desquamation. If possible the palpable tumour masses should receive treatment boosts.

\section{Surgery}

Mastectomy is generally not indicated in the presence of features of locally advanced disease, but the role of surgery is changing. Aggressive treatment with a combination of cytotoxic drugs or initial hormonal treatment often causes the primary tumour to regress to a lower stage (with disappearance of peau d'orange and erythema and reduction in tumour volume), making surgery feasible some weeks or months after the start of systemic treatment. In such cases surgery may be a wide excision and clearance of axillary nodes or a total mastectomy and node clearance, both being followed by radiotherapy to the remaining breast or to the chest wall.
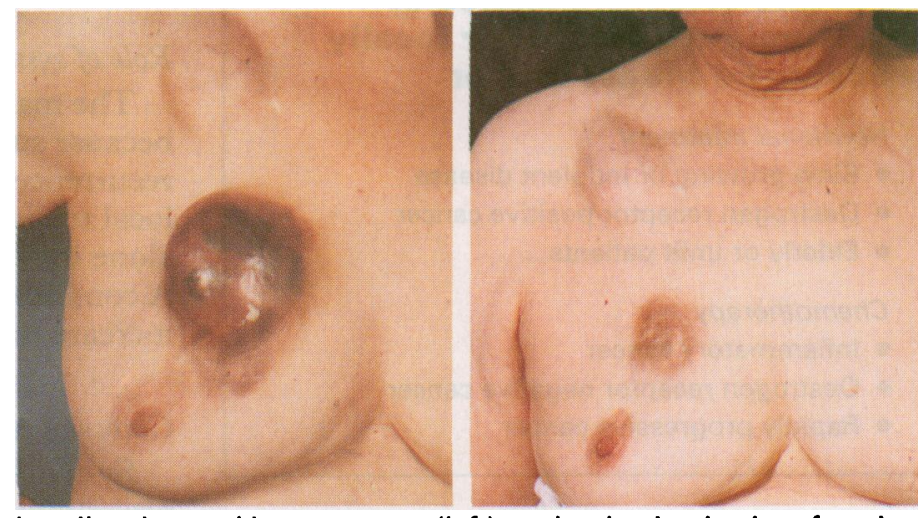

Locally advanced breast cancer (left); and reduction in size after six months of tamoxifen treatment (right). The mass in the infraclavicular region is a lipoma. 


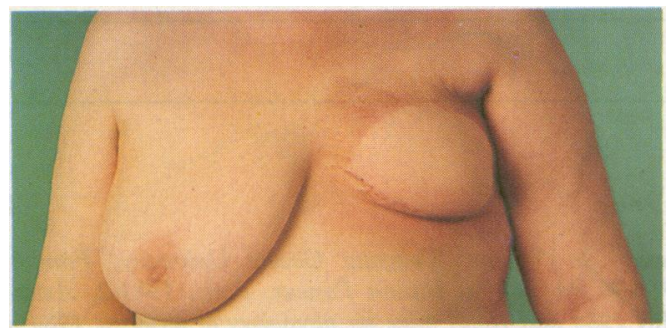

Salvage mastectomy and coverage with myocutaneous flap (from latissimus dorsi) for treatment of residual disease after chemotherapy and radiotherapy.

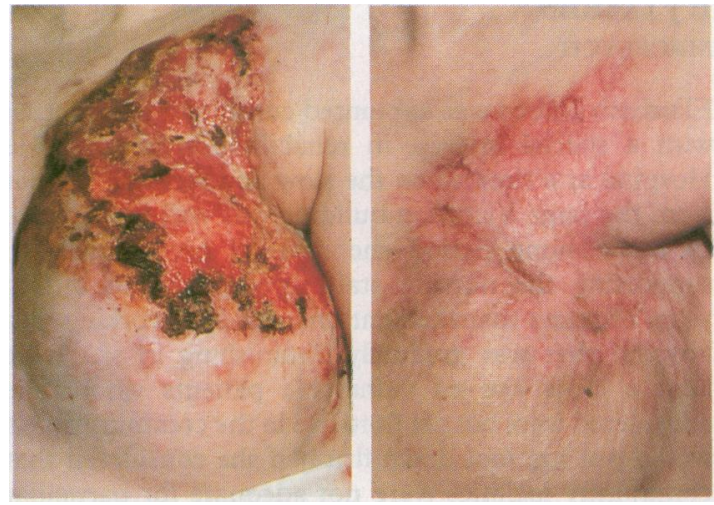

Locally advanced breast cancer with ulceration (left); and good response and re-epithelialisation after three courses of intra-arterial chemotherapy (right).
In some patients residual disease remains in the breast despite systemic treatment and radiotherapy. This disease can be excised by a salvage mastectomy, ideally followed by coverage with a myocutaneous flap (from latissimus dorsi or transverse rectus abdominus). "Toilet" surgery, used in an effort to control fungating cancers or recurrence and progression of disease, is often ineffective and should only be performed for breast cancers that are locally advanced either because of their peripheral position in the breast or because of a delay in presentation. In this group surgery should be combined with radiotherapy and appropriate adjuvant systemic treatment.

\section{Intra-arterial chemotherapy}

Despite the best efforts with combined treatments, a substantial proportion of patients who present with locally advanced disease develop uncontrolled disease of the chest wall. Although low dose intravenous chemotherapy by infusion (for example, infusional fluorouracil) can relieve symptoms in up to half of these patients, the overall efficacy of systemic chemotherapy is poor. Because of technical difficulties, investigation of intra-arterial chemotherapy has been limited to uncontrolled studies in a few centres. However, the best published series report impressive response rates with low toxicity in patients presenting initially with locally advanced breast cancer. If intra-arterial treatment does not produce a response after the first course it is probably not worth pursuing. The drugs and doses used are similar to those given intravenously for palliative treatment. One problem with delivery of drugs by the intra-arterial route is that in patients who have received radiotherapy the blood vessels supplying the chest wall may be damaged, resulting in an impaired blood supply that limits drug delivery.

\section{Local recurrence after mastectomy}

\section{Treatment of local recurrence in chest wall}

\begin{tabular}{|c|c|}
\hline $\begin{array}{l}\text { Type of } \\
\text { recurrence }\end{array}$ & Treatment \\
\hline Single spot & $\begin{array}{l}\text { Excise and consider } \\
\text { radiotherapy }\end{array}$ \\
\hline $\begin{array}{l}\text { Multiple } \\
\text { spot }\end{array}$ & $\begin{array}{l}\text { Radiotherapy unless already } \\
\text { given or more radical excision } \\
\text { (possibly with coverage with } \\
\text { myocutaneous flap) }\end{array}$ \\
\hline Widespread & $\begin{array}{l}\text { Consider radiotherapy unless } \\
\text { already given or disease too } \\
\text { widespread } \\
\text { Appropriate systemic treatment } \\
\text { (hormonal or chemotherapy) } \\
\text { Intra-arterial chemotherapy } \\
\text { Intravenous infusion of } \\
\text { fluorouracil }\end{array}$ \\
\hline
\end{tabular}

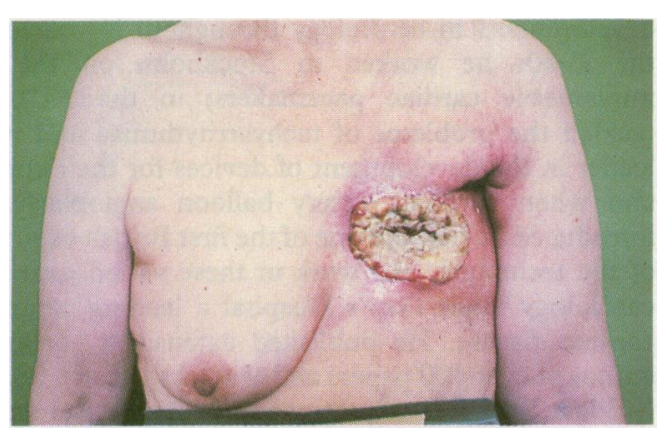

Longstanding, isolated, large, unsightly, and malodorous local recurrence after mastectomy and radiotherapy.
This usually occurs in the skin flaps adjacent to the scar and is presumed to arise from viable cells shed during surgery. It can usually be diagnosed by fine needle aspiration cytology. Local disease can be isolated, but in up to half of patients it heralds systemic relapse. For this reason a search for distant metastases should be undertaken in all patients.

Local recurrence after mastectomy can be classified as single spot relapse, multiple spot relapse, or field change. Treatment and prognosis differ for these three categories.

\section{Treatment}

If the recurrence is focal and occurs many years after the original surgery excision alone can provide long term control. If the recurrence is not single but still localised then the options are radiotherapy or a more radical excision. In a more widespread recurrence standard treatments are often disappointing, although intra-arterial chemotherapy and infusional fluorouracil are sometimes effective. Failure to halt the progress of local disease can lead to cancer en cuirasse-where the chest wall is encircled by tumour-a most unpleasant situation for the patient.

Recurrence in the chest wall can be quite indolent, grow slowly, and occur in the absence of metastases elsewhere. The control of ulceration and focal malodorous infected tissue is a considerable problem for carers, and patients with such disease have a miserable existence. Excision of dead tissue and the use of topical and oral antibiotics with antianaerobic activity combined with charcoal dressings help to control the malodour. The best form of treatment is prevention by ensuring that initial local treatment is optimal.

J M Dixon acknowledges the support of the Cancer Research Campaign. Photographs of the patient treated by intra-arterial chemotherapy were provided by Mr J R C Sainsbury, consultant surgeon at Huddersfield Royal Infirmary. Other photographs were prepared by $\mathrm{Mr} \mathrm{D}$ Dirom, Medical Illustration Unit, University of Edinburgh.

A Rodger is director of William Buckland Radiotherapy Centre at Alfred Hospital and professor of radiation oncology at Monash University, Melbourne, Australia; R C F Leonard is consultant medical oncologist at Department of Clinical Oncology, Western General Hospital, Edinburgh; and J M Dixon is senior lecturer in surgery at Edinburgh Royal Infirmary and honorary consultant surgeon at Western General Hospital, Edinburgh.

The ABC of Breast Diseases has been edited by J M Dixon. 